Supplement of Geosci. Model Dev., 13, 841-858, 2020

https://doi.org/10.5194/gmd-13-841-2020-supplement

(c) Author(s) 2020. This work is distributed under

the Creative Commons Attribution 4.0 License.

(c) (1)

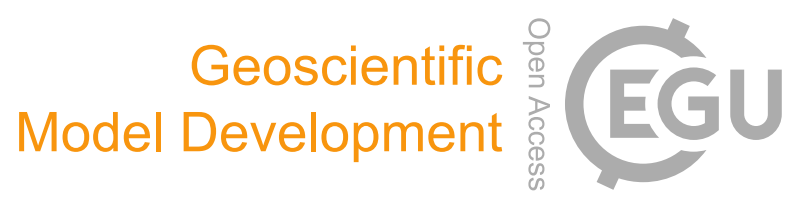

Supplement of

\title{
Reconstructing climatic modes of variability from proxy records using ClimIndRec version 1.0
}

Simon Michel et al.

Correspondence to: Simon Michel (simon.michel@u-bordeaux.fr)

The copyright of individual parts of the supplement might differ from the CC BY 4.0 License. 


\section{Supplementary table 1: Proxy records not in PAGES 2k}

\begin{tabular}{|c|c|c|c|c|c|c|c|c|c|c|c|}
\hline $\mathrm{N}^{\circ}$ & Code & Location & Longitude $\left({ }^{\circ} \mathrm{E}\right)$ & Latitude $\left({ }^{\circ} \mathrm{N}\right)$ & First year & Last year & Archive & Proxy type & Related variable & Seasonality & Ref. \\
\hline 1 & accr-crete-yr-annual & Crete & -38.50 & 71.00 & 1000 & 1973 & Ice core & Snow accumulation & Precip. & Annual & Andersen et al. 2006 \\
\hline 2 & accr-GISP2-yr-annual & GISP2 & -38.50 & 72.60 & 1000 & 1988 & Ice core & Snow accumulation & Precip. & Annual & Cuntever all. 1995 \\
\hline 3 & d180-agass-79-annual & Agassiz & -77.00 & 80.70 & 1000 & 1972 & Ice core & & SAT & Annual & Fisher etall \\
\hline 4 & icecore-GISP2-ssNA-annual & GISP2 & -39.00 & 73.00 & 1000 & 1986 & Ice core & Sea salt $\mathrm{Na}$ & SLP & DJF & Nivecker and Mavewski. 2002 \\
\hline 5 & speleo-crystal-d18O-annual & Crystal cave & -121.00 & 36.90 & 1000 & 2007 & peleothem & & SAT & Annual & Mccabe-Givanetan, 2013 \\
\hline 6 & tree-alps-Tijas-annual & European Alps & 9.00 & 46.00 & 1000 & 2004 & Tree ring & Tree ring MXD & SAT & $\mathrm{JJAS}$ & Dunten erall. zO1Z \\
\hline 7 & tree-AR050-stah-annual & Black Swamp & -91.30 & 35.15 & 1019 & 1980 & ree ring & Tree ring width & SAT & Annual & Stanie 1996a \\
\hline 8 & tree-AR052-stah-annual & Mayberry Slough & -89.00 & 35.50 & 1000 & 1990 & ree ring & Tree ring width & SAT & Annual & stane ana creaveland, 2005a \\
\hline 9 & tree-CA051-tosh-annual & San Gorgonio & -116.82 & 33.40 & 1000 & 1970 & ree ring & Tree ring width & SAT & Annual & Iosn 1994 \\
\hline 10 & tree-ca605-king-annual & Mammoth Peak & -119.00 & 41.00 & 1000 & 1996 & Tree ring & Tree ring width & Precip. & NDJFM & Dumnerall 2005 \\
\hline${ }_{12}^{11}$ & $\begin{array}{l}\text { tree-CA640-graum-annual } \\
\text { tree-c557-woodho-annuaut }\end{array}$ & $\begin{array}{l}\text { Hamilton } \\
\text { Lily Lake }\end{array}$ & -118.92 & 39.00 & 1000 & 1988 & Tree ring & Tree ring width & $\begin{array}{l}\text { Precip. } \\
\text { SAT. }\end{array}$ & NDJFM & Bunnetall zous \\
\hline${ }_{13}^{12}$ & $\begin{array}{l}\text { tree-co572-woodho-annual } \\
\text { tree-Fro0-1-tha-2nnwal }\end{array}$ & $\begin{array}{r}\text { Lily Lake } \\
\text { Choctawhatche River }\end{array}$ & -105.60 & 40.30 & 1000 & 1998 & Tree ring & Tree ring width & SAT & Annual & woounouse ana Brown. 2006 \\
\hline $\begin{array}{l}13 \\
14\end{array}$ & $\begin{array}{l}\text { tree--L0001-stah-annnal } \\
\text { tree-forfio-cloud-annual }\end{array}$ & $\begin{array}{l}\text { Choctawhatchee River } \\
\text { Forfoiorddalen }\end{array}$ & -85.92 & 30.45 & 1000 & ${ }_{2902}^{1992}$ & Tree ring & $\begin{array}{l}\text { Tree ring width } \\
\text { Tree ring } \delta^{13} \mathrm{C}\end{array}$ & SAT & Annual & \\
\hline 15 & 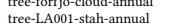 & $\begin{array}{l}\text { Forjuofadalan } \\
\text { Big Cypress }\end{array}$ & $\begin{array}{l}15 . / 3 \\
-9297\end{array}$ & 32.80 & ${ }_{1000}^{1000}$ & ${ }_{1988}^{2001}$ & $\begin{array}{l}\text { Tree ring } \\
\text { Tree ring }\end{array}$ & $\begin{array}{l}\text { Tree ring } \delta^{5.5} \mathrm{C} \\
\text { Tree ring width }\end{array}$ & $\begin{array}{l}\text { Cloud \% } \\
\text { SAT }\end{array}$ & JA & 10ung e tall zoll \\
\hline 16 & tree-mor-pdsi-annual & Morocco & -5.00 & 33.75 & 1049 & 2001 & $\begin{array}{l}\text { Iree ring } \\
\text { Tree ring }\end{array}$ & $\begin{array}{l}\text { Iree ring wiath } \\
\text { Tree ring width }\end{array}$ & $\begin{array}{l}\text { SAI } \\
\text { PSI }\end{array}$ & $\begin{array}{l}\text { Annual } \\
\text { FMAMI }\end{array}$ & Stanie $1996 \mathrm{~b}$ \\
\hline 17 & tree-mt113-wagon-annual & Yellow Mountain Ridge (Entire Bark Trees) & -109.80 & 45.60 & 1000 & 1998 & ree ring & Tree ring width & Precip. & 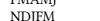 & Graumlichet al 2003 \\
\hline 18 & tree-NM584-touch-annual & Mesa Alta & -106.60 & 36.20 & 1000 & 2007 & ree ring & Tree ring width & $\begin{array}{l}\text { Preccp. } \\
\text { Precip. }\end{array}$ & ONDIFMAMI & Graumlich et al. 2003 \\
\hline 19 & tree-NV516-grayb-annual & Hill 10842 & -114.20 & 38.90 & 1000 & 1984 & ree ring & Tree ring width & SAT & Annual & 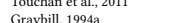 \\
\hline 20 & tree-nv517-grayb-annual & Spring Mountains Lower & -114.70 & 34.30 & 1000 & 1984 & ree ring & Tree ring width & SAT & Annual & Gravbill \\
\hline 21 & tree-SCpla-precip-annual & S. Colorado Plateau I & -109.30 & 37.50 & 00 & 1987 & ree ring & Tree ring width & Precip. & ctober-July & Gravber and N1pmueller. 2005 \\
\hline 22 & tree-S & S. Colorado Plate & -110.70 & 36.50 & 00 & 96 & ing & Tres & SAT & Annual & Salzer and Kidrmueller, 2005 \\
\hline 23 & tree-siber-temp-annual & Taimyr-Putoran & 103.00 & 71.29 & 00 & 96 & ee ring & Tree ring width & AT & Annual & INaurzDaev e al, zovz \\
\hline 24 & tree-s & Laue & 50 & 46.42 & 100 & 76 & ing & th & & JA & Schweingruber. 1988 \\
\hline 25 & tree-U & Id Horse Ridge & -110.10 & 40.00 & 1000 & 35 & ing & Ith & & nual & Graybill 1994c \\
\hline 26 & tree-U1 & immoth & -112.67 & 37.65 & 1000 & 1989 & ree ring & Tree & $\mathrm{s}$ & Annual & Gravbull IY94a \\
\hline 27 & tree-all & Albermale Sound & & & 934 & 05 & ing & vidth & PDSI & & stance ex at. 2013 \\
\hline 28 & tree-arj] & Arjeplog & 17.90 & 66.50 & 1200 & 2010 & ee ring & Tree & SAT & & Brorkiunde ala. 20 14 \\
\hline 29 & -mxd-annual & Jtantland & 15.00 & 63.10 & 800 & 2011 & ing & & $\mathrm{s}$ & & Znang er all zolo \\
\hline${ }_{31}^{30}$ & 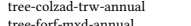 & $\begin{array}{l}\text { Col du Zad } \\
\text { orfiorffalen-x }\end{array}$ & $\begin{array}{l}-5.10 \\
15.70\end{array}$ & 33.00 & 984 & $\begin{array}{l}1984 \\
2005\end{array}$ & ee ring & dth & $\mathrm{PS}$ & FMAMJ & Esper et al...2001 \\
\hline $\begin{array}{l}31 \\
32\end{array}$ & $\begin{array}{l}\text { tree-fort-mmd-annual } \\
\text { tree-khibiny-bi-annual }\end{array}$ & $\begin{array}{l}\text { Forf jorrtalen- } x \\
\text { Khibiny }\end{array}$ & $\begin{array}{l}15.70 \\
33.50\end{array}$ & 68.80 & $\begin{array}{l}978 \\
821\end{array}$ & 2005 & $\begin{array}{l}\text { ree ring } \\
\text { ree ring }\end{array}$ & $\begin{array}{l}\text { Tree ring MXD } \\
\text { Tree ring BI }\end{array}$ & $\begin{array}{l}\text { SAT } \\
\text { SAT }\end{array}$ & $\begin{array}{l}\text { AMAJAS } \\
\text { JIA }\end{array}$ & 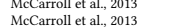 \\
\hline 33 & tree-laanila-mxd-annual & ila & 27.30 & 68.50 & 800 & 2005 & Tree ring & Tree ring MXD & SAT & $\mathrm{JIA}$ & Niccarroll etal. ZU13 \\
\hline 34 & tree-1 & $\mathrm{SM}_{\mathrm{a}}$ & -97.10 & 49.50 & 1409 & 1998 & Tree ring & Tree ring width & & Annual & George and Nielesen zoo2 \\
\hline 35 & annual & Mesoan & -100.00 & 20 & 800 & 2008 & & Tre & & June & Dtante etal. zo11 \\
\hline 36 & accr- & & & & 800 & 1995 & re & ulation & & Annual & Andersen et al. zo07 \\
\hline 37 & & Potom & & & 950 & & & & Stream flow & & Maxwell et al. zon \\
\hline 38 & & & -77 & & 1373 & & & & & MJJAS & Not rouna) \\
\hline 39 & & & & & 950 & & & & & iJJ & Wilson et all 2013 \\
\hline 40 & & & & & 800 & & & acrement & & 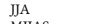 & Linanom ana Jakkanen. 2011 \\
\hline 41 & & Southern Fi & & & 800 & & & & & MJJAS & Helama et al. 2014 \\
\hline 42 & tree-s & V Tu & & 37.00 & 1339 & 19 & & Tree & Precip. & & atis 2003 \\
\hline 43 & tree-tyrol-mxd-annual & & 12.50 & 48.00 & 1053 & & & Tree ring MXD & & & Esper etal \\
\hline 44 & d180-NIshelf-yr-annual & North Icelandic Shelf & 66.53 & -18.20 & 953 & 2000 & Ice core & & SAT & Annual & Reynolds et al., 2016 \\
\hline
\end{tabular}


Supplementary table 2: Ten largest volcanic eruptions from Ortega et al., 2015

\begin{tabular}{rll}
\hline Volcano & Country & Date \\
\hline Unknown & & 1229 \\
Samalas & Indonesia & 1257 \\
Unknown & & 1285 \\
Huaynaputina & Peru & 1600 \\
Parker & Phillippines & 1640 \\
Serua & Indonesia & 1693 \\
Unknown & & 1809 \\
Tambora & Indonesia & 1815 \\
Cosiguina & Nicaragua & 1834 \\
Krakatau & Indonesia & 1883 \\
\hline
\end{tabular}


Supplementary table 3: Eleven largest volcanic eruptions from Sigl et al., 2015

\begin{tabular}{rll}
\hline Volcano & Country & Date \\
\hline Unknown & & 1108 \\
Unknown & & 1171 \\
Unknown & & 1230 \\
Samalas & Indonesia & 1258 \\
Kuwae & Vanuatu & 1458 \\
Huaynaputina & Peru & 1601 \\
Parker & Phillippines & 1641 \\
Serua & Indonesia & 1695 \\
Lakagigar & Iceland & 1783 \\
Unknown & & 1809 \\
Tambora & Indonesia & 1815 \\
\hline
\end{tabular}




\section{Supplementary table 4: Variance of the NAO}

\begin{tabular}{rcccc}
\hline Method & Mean test variance [range] & Mean train variance [range] & Variance 1000-1855 & Variance 1856-1970 for Enet and PCR (1856-1972 for RF and PLS) \\
\hline RF & $0.24[0.08,0.47]$ & $0.74[0.64,0.84]$ & 0.16 & 0.72 \\
Enet & $0.26[0.09,0.96]$ & $0.4[0.27,0.7]$ & 0.18 & 0.33 \\
PCR & $0.35[0.06,0.77]$ & $0.56[0.37,0.86]$ & 0.56 & 0.62 \\
PLS & $0.33[0.08,1.04]$ & $0.57[0.4,0.8]$ & 0.17 & 0.43 \\
NAO (1000-1970) & $1.44[1.23,1.61]$ & $1.46[0.72,2.27]$ & 1.44 & 1.46 \\
NAO (1000-1973) & $1.44[1.27,1.57]$ & $1.57[0.73,2.33]$ & & \\
\hline
\end{tabular}




\section{Supplementary table 5: R packages used in ClimIndRec}

\begin{aligned} & \hline Package Reference \\ & \hline glmnet Friedman et al. 2010 \\ & pls Mevik et al., 2007 \\ & randomForest Liaw and Wienner 2002 \\ & ncdf4 Pierce, 2017 \\ & stringr Wickham 2017 \\ & \hline\end{aligned}




\section{Supplementary material 1: Regression methods}

We present each method in two steps: model fitting (for training) and reconstruction (for testing). For each method the proxy matrix is denoted as $X \in \mathbb{R}^{n \times p}$ the proxy predictor set and the climate index as $Y \in \mathbb{R}^{n}$. $X_{(r e c)} \in \mathbb{R}^{m \times p}$ is the testing dataset from which a $\mathbb{R}^{m}$ reconstruction is build using the regression method

\subsection{Principal Component Regression (PCR)}

\subsubsection{Modeling}

The Principal Component Regression (Hotelling, 1957) method consists in finding the best linear combination between $Y$ and the Principal Component of $X$. The Principal Component Analysis (PCA) consists in applying an orthogonal transformation of an initial set of variables, potentially correlated between them, into another set of linearly uncorrelated variables: the Principal Component (Pearson, 1901; Hotelling, 1933).

The first step consists in building an orthogonal basis where $X$ will be projected. We define $S \in \mathbb{R}^{p \times p}$, as the empirical estimator of the covariance matrix of $X$ :

$$
S=\frac{1}{n} X^{T} X \in \mathbb{R}^{p \times p}
$$

We calculate the orthogonal basis formed by the vectors $v_{1}, \ldots, v_{p}$ by diagonalizing $S$ :

$$
\begin{gathered}
v_{1}=\arg \max _{\substack{v \in \mathbb{R}^{p} \\
\|v\|=1}} v^{T} S v \\
v_{2}=\arg \max _{\substack{v \in \mathbb{R}^{p} \\
\|v\|=1 \\
\left\langle v^{T} v_{1}\right\rangle=0}} v^{T} S v \\
\cdots \\
v_{p}=\arg \max _{\substack{v \in \mathbb{R}^{p} \\
\|v\|=1 \\
\left\langle v^{T} v_{1}\right\rangle=0 \\
\left\langle v^{T} v_{p-1}\right\rangle=0}} v^{T} S v \\
\left\langle v^{2}\right\rangle
\end{gathered}
$$

where $\|v\|=\sqrt{\sum_{j=1}^{p}\left(v^{j}\right)^{2}}, \forall v \in \mathbb{R}^{p}$. It is equivalent to maximizing step by step the empirical variance of the projection of $X$ on each orthogonal axis. Indeed, $\forall v \in \mathbb{R}^{p}$ :

$$
v^{T} S v=\frac{1}{n-1} v^{T} X^{T} X v=\frac{1}{n-1}(X v)^{T}(X v)=\operatorname{Var}_{e m p}(X v)
$$

The vectors $\left(v_{k}\right)_{1 \leq k \leq p}$ are called the Empirical Orthogonal Functions (EOFs). It corresponds to the eigenvectors of the covariance matrix and each contains a given part of the spatial variability of the proxy dataset. We attribute them the eigenvalues $\left(\lambda_{k}\right)_{1 \leq k \leq p}$, which corresponds to the initial variance of $X$ translated by each orthogonal projection in the new basis:

$$
\lambda_{k}=\operatorname{Var}\left(X v_{k}\right)=v_{k}^{T} S v_{k} \quad \forall 1 \leq k \leq p
$$


The Principal Components $\left(u_{1}, \ldots, u_{p}\right)$ are then the projections of $X$ on the EOFs. We denote $V=\left(v_{1}, \ldots, v_{p}\right)$. We then calculate the Principal Component matrix $U=\left(u_{1}, \ldots, u_{p}\right)$, defined as:

$$
U=X V \in \mathbb{R}^{n \times p}
$$

Now, we regress $Y$ on the $q \leq p$ (see subsection 3.1.3) first Principal Component. These $q$ Principal Component are merged in a submatrix of $U: \mathcal{U}=\left(u_{k}\right)_{1 \leq k \leq q}$. The model is given by:

$$
Y=\mathcal{U} \beta+\epsilon
$$

Where $\epsilon$ is a white noise vector of size n.

The best estimator for $\beta=\left(\beta_{1}, \ldots, \beta_{q}\right)$, is given by the Ordinary Least Squares (OLS) estimator which minimizes $\|\hat{\epsilon}\|=\|Y-\hat{Y}\|:$

$$
\hat{\beta}_{O L S}=\left(\mathcal{U}^{T} \mathcal{U}\right)^{-1} \mathcal{U}^{T} Y
$$

\subsubsection{Reconstruction}

We project the testing matrix $X_{(r e c)}$ on the pre-calculated orthogonal basis $V$ :

$$
U_{(r e c)}=X_{(r e c)} V \in \mathbb{R}^{m \times p}
$$

We then obtain the reconstruction by applying the estimated coefficient vector on the sub-matrix $\mathcal{U}_{(r e c)}=$ $\left(U_{(r e c)}^{1}, \ldots, U_{(r e c)}^{q}\right) \in \mathbb{R}^{m \times q}$ :

$$
\hat{Y}_{q}=\mathcal{U}_{(r e c)} \hat{\beta}_{O L S} \in \mathbb{R}^{m}
$$

\subsubsection{Control parameters}

Here, $q$ is the unique control parameter to be tuned. Here the control parameter vector $\theta$ is unidimensional and takes its values in the discrete set $\{i\}_{1 \leq i \leq p}$ and its tuning is obtained by KFCV.

\subsection{Partial Least Squares Regression}

The PCA keeps most of the initial variance in $X$ in a lower number of vectors. But EOFs $v_{1}, \ldots, v_{p}$ are constructed without taking into account any information about the predictand $Y$. Another possible approach is thus to determine the orthogonal basis in which the empirical covariance between $Y$ and the projection of $X$ on that former is maximized. This is the Partial Least Squares regression (PLSr) method (Wold, 1983).

The first latent variable (LV), denoted $\xi_{1}=\sum_{j=1}^{p} v_{1, j} X^{j}=X v_{1}$, where $X \in \mathbb{R}^{n \times p}$ and $v_{1} \in \mathbb{R}^{p}$ is the linear combination of the initial variables $X^{1}, \ldots, X^{p}$ such as:

$$
v_{1}=\arg \max _{\substack{u \in \mathbb{R}^{p} \\\|v\|=1}} \operatorname{Cov}(Y, X v),
$$


In a similar approach to the PCR, the second LV is $\xi_{2}=\sum_{j=1}^{p} v_{2, j} X^{j}=X v_{2}$, orthogonal to $\xi_{1}$, such as:

$$
v_{2}=\arg \max _{\substack{v \in \mathbb{R}^{p} \\\|v\|=1 \\\left\langle\xi^{1}, X v\right\rangle=0}} \operatorname{Cov}(Y, X v)
$$

And so on, until we have $r \leq p$ LVs. The LV matrix is denoted $\Xi=\left[\xi_{1}, \ldots, \xi_{p}\right]$. Here, $v_{1}, \ldots, v_{p} \in \mathbb{R}^{p}$, are analogous to the EOFs in PCA, and are here called loadings. The latent variables $\xi_{1}, \ldots, \xi_{r}$ respectively correspond to the projection of $X$ on the $r$ loadings.

Finding the loadings is not as trivial as for PCR. Indeed the empirical covariance matrix is not necessary definite positive and thus cannot be diagonalized. We solve this problem by using the algorithm 1 named PLS1. Analogously to the PCR, the method provides various alternative reconstructions depending on the value of $r$, which corresponds to the number of LVs kept as regressors.

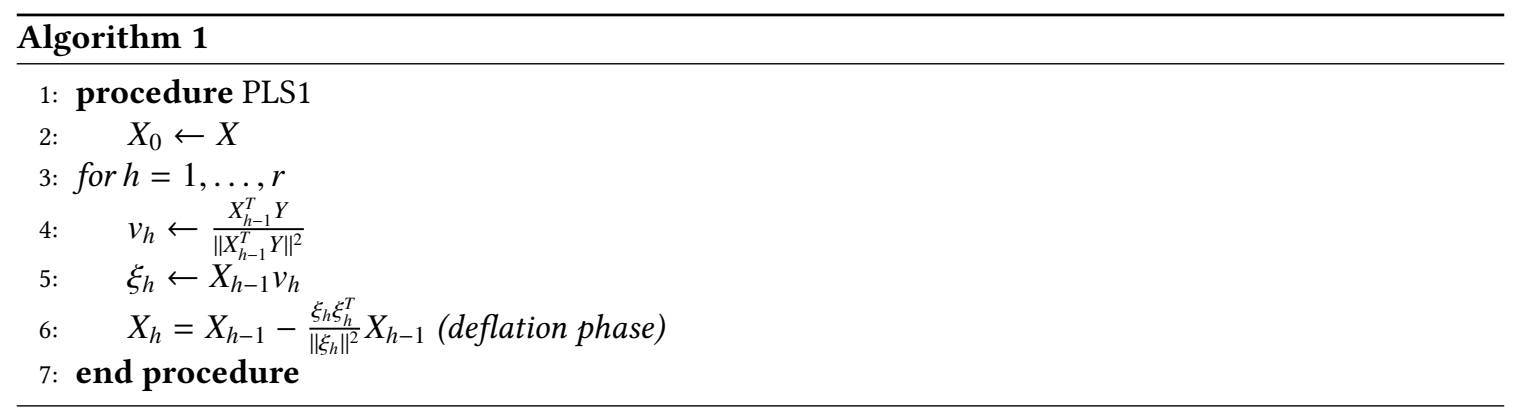

Now we regress $Y$ on the $r \leq p$ first LVs. These $r$ LVs are merged in a submatrix of $\Xi: \Psi=\left(\xi_{k}\right)_{1 \leq k \leq r}$. The model is given by:

$$
Y=\Psi \beta+\epsilon
$$

Where $\epsilon$ is a white noise vector of size n.

The best estimator for $\beta=\left(\beta_{1}, \ldots, \beta_{q}\right)$, is given by the Ordinary Least Squares (OLS) estimator which minimizes $\|\hat{\epsilon}\|=\left\|Y-\hat{Y}_{q_{K F}}\right\|$ :

$$
\hat{\beta}_{O L S}=\left(\Psi^{T} \Psi\right)^{-1} \Psi^{T} Y
$$

\subsubsection{Reconstruction}

The reconstruction is done in the same way as for PCR. Using the first seen data matrix $X_{(\text {rec })}$ (section 2.4), we project the latter on the pre-calculated orthogonal basis $V$ :

$$
\Xi_{(r e c)}=X_{(r e c)} V \in \mathbb{R}^{m \times p}
$$

The reconstruction is obtained by applying the estimated coefficient vector on the sub-matrix $\Psi_{(\text {rec })}=$ $\left(\xi_{(r e c)}^{1}, \ldots, \xi_{(r e c)}^{r}\right) \in \mathbb{R}^{m \times r}$ :

$$
\hat{Y}_{r}=\Psi_{(r e c)} \hat{\beta}_{O L S} \in \mathbb{R}^{m}
$$




\subsubsection{Control parameters}

For the PLSr method, $r$ is the unique control parameter to be tuned. Analogously to the Principal Component Analysis, the tuning of that latter is obtained by KFCV.

\subsection{Elastic Net regression}

\subsubsection{Modeling}

Without using orthogonal transformation of the initial variables as in PCR and PLSr, the most simple predictive model is the multiple linear regression model:

$$
Y=X^{1} \beta_{1}+\cdots+X^{p} \beta_{p}+\epsilon
$$

Where $\epsilon \sim \mathcal{N}\left(0, \sigma^{2}\right)$ and $\operatorname{Cov}\left(\epsilon_{i}, \epsilon_{j}\right)=0$ if $i \neq j$.

The reconstruction of $Y$, given $p$ proxy records $X^{1}, \ldots, X^{p}$ is obtained by the equation:

$$
\hat{Y}=X^{1} \hat{\beta}_{1}+\cdots+X^{p} \hat{\beta}_{p}
$$

$\hat{\beta}=\left(\hat{\beta}_{1}, \ldots, \hat{\beta}_{p}\right)$ are the regression coefficients, which are obtained by the OLS predictor. However, this usual regression model is known to often result in a poor reconstruction accuracy due to the several assumptions made on the original data (Poole, 1971), which are often not verified, such as homoscedasticity and errors normality. Several studies developed regularized (or penalized) regression methods to overcome the OLS defaults. Here we focus on the Elastic Net regression (Zou and Hastie, 2005), which is a combination of the Ridge regression (Hoerl, 1970) and the Lasso regression (Tibshirani et al., 1996). All these methods have been developed to avoid the high variability of the OLS predictor when the number of predictors is relatively high. The Ridge regression shrinks towards zero the estimated coefficients associated to predictors unlinked to the predictand. No predictor selection is made by this method, but the shrunken estimated coefficients modulate the importance of these in the model. By contrast, the Lasso also reduces the variability of the estimates, but in this case by shrinking to zero the estimated coefficients associated to unreliable variables. Hence, a selection is made by rejecting variables associated to coefficients shrunk to zero.

The idea of a regularized (or penalized) regression is to add a threshold constraint using the $l_{k}$ norm of $\beta$ : $\|\beta\|_{k}^{k}=\sum_{j=1}^{k}\left|\beta_{j}\right|^{k}$. With $k=1$ in Lasso regression, and $k=2$ in Ridge regression. The penalized loss functions are given by:

$$
\begin{aligned}
& L^{\text {ridge }}(\beta)=\left\|Y-\sum_{j=1}^{p} \beta_{j} X^{j}\right\|^{2}+\lambda_{2} \sum_{j=1}^{p} \beta_{j}^{2} \\
& L^{\text {lasso }}(\beta)=\left\|Y-\sum_{j=1}^{p} \beta_{j} X^{j}\right\|^{2}+\lambda_{1} \sum_{j=1}^{p}\left|\beta_{j}\right| \\
& L^{\text {enet }}(\beta)=\left\|Y-\sum_{j=1}^{p} \beta_{j} X^{j}\right\|^{2}+\lambda_{1} \sum_{j=1}^{p}\left|\beta_{j}\right|+\lambda_{2} \sum_{j=1}^{p} \beta_{j}^{2}
\end{aligned}
$$

$\lambda_{1}$ penalizes the sum of the absolute values of the regression coefficients while $\lambda_{2}$ penalizes their summed squares. Here, $\lambda_{1}, \lambda_{2}>0$. 
Let $w=\left(w_{j}\right)_{1 \leq j \leq p}=\left(\operatorname{sgn}\left(\beta_{j}\right)\right)_{1 \leq j \leq p}$, where sgn is the sign function. The loss functions can then be denoted as:

$$
\begin{aligned}
& L^{\text {ridge }}=\|Y-X \beta\|^{2}+\lambda_{2} \beta^{T} \beta \\
& L^{\text {lasso }}=\|Y-X \beta\|^{2}+\lambda_{1} w^{T} \beta \\
& L^{\text {enet }}=\|Y-X \beta\|^{2}+\lambda_{1} w^{T} \beta+\lambda_{2} \beta^{T} \beta
\end{aligned}
$$

The estimated regression coefficients obtained by minimizing the Lasso and the Ridge loss functions are:

$$
\begin{aligned}
& \hat{\beta}^{\text {lasso }}=\left(X^{T} X\right)^{-1}\left(X^{T} Y-\frac{\lambda_{1}}{2} w\right) \\
& \hat{\beta}^{\text {ridge }}=\left(X^{T} X+\lambda_{2} I\right)^{-1} X^{T} Y
\end{aligned}
$$

The Elastic Net regression coefficients are then estimated by minimizing $L^{\text {enet }}$ :

$$
\hat{\beta}^{\text {enet }}=\left(X^{T} X+\lambda_{2} I\right)^{-1}\left(X^{T} Y-\frac{\lambda_{1}}{2} w\right)
$$

An alternative way to write this equation as a linear combination of $\hat{\beta}^{\text {lasso }}$ and $\hat{\beta}^{\text {ridge }}$ is:

$$
\hat{\beta}^{\text {enet }}=\left(X^{T} X+(1-\alpha) \lambda I\right)^{-1}\left(X^{T} Y-\frac{\alpha \lambda}{2} w\right)
$$

where $\alpha \in[0,1]$. If $\alpha=1$, a Rigde regression is applied, and if $\alpha=0$, we apply a Lasso regression.

\subsubsection{Reconstruction}

The reconstruction is obtained by applying the estimated regression coefficients $\hat{\beta}^{\text {enet }}$ on the validation variables $X_{v a l}^{1}, \ldots, X_{v a l}^{p}$ :

$$
\hat{Y}_{\lambda, \alpha}=\sum_{j=1}^{p} X_{(v a l)}^{j} \hat{\beta}_{j}^{\text {enet }}
$$

\subsubsection{Hyperarameters}

For Enet method, the control parameters are $\lambda$ and $\alpha$ such that $\theta=(\alpha, \lambda)$. The latter controls the relative balance between the Lasso and Ridge regularization, while the former controls the overall intensity of regularization as $\lambda_{1}$ (resp. $\lambda_{2}$ ) in Lasso (resp. Ridge regularization). A high $\alpha$ suggests a dense model with many but small non-zero coefficients. A low $\alpha$ suggests a sparse model with many zero coefficients. In our case, since we want a general methodology performant for each random split, we apply two simultaneous and nested KFCV to find the best estimated pair $(\hat{\lambda}, \hat{\alpha})$.

Since $\lambda$ and $\alpha$ take respectively their values in the continuous sets $\mathbb{R}^{p}$ and $[0,1]$, we have to discretize their respective intervals for the parameter estimation. The finer these discretizations are, the more reliable the parameters will be, at the expanse of the computational time. In the actual version of ClimIndRec (version 1.0) the set where $\alpha$ is tuned is $(0.1 i)_{0 \leq i l e q 10}$ and the set where $\lambda$ is tuned is $(0.2 i)_{0 \leq i \leq 25}$. It can be easily changed by modifying the code main R script of ClimIndRec. 


\subsection{Random Forest regression}

The random forest has been introduced by Breiman (2001) as a learning method for regression. The method relies on using randomization to minimize the reconstruction uncertainty given by regression trees. Random forests encompass a large variety of regression methods (Breiman, 2001). Here, we present the most classical kind of random forests known as random-input random forests (Breiman, 2001).

\subsubsection{Modeling}

First we have to define regression trees. We denote each set of predictand/predictors by $\left.\left\{Y_{i}, X_{i}\right)_{1 \leq i \leq n}\right\}$ where $X_{i}=\left(X_{i}^{1}, \ldots, X_{i}^{p}\right)$, is the ensemble of proxy records for the $i^{\text {th }}$ time step, and $Y_{i}$ the corresponding values of the climate index at the same time step, $\forall 1 \leq i \leq p$. All the observations, $\left.\left\{Y_{i}, X_{i}\right)_{1 \leq i \leq n}\right\}, \forall 1 \leq i \leq p$, are put on the root of the tree. The first step consists in cutting that root in two child nodes. A cut is defined as:

$$
\left\{X^{j} \leq d\right\} \cup\left\{X^{j} \geq d\right\}
$$

where $j=\{1, \ldots, p\}$ and $d \in \mathbb{R}$. Cutting a node with $\left\{X^{j} \leq d\right\} \cup\left\{X^{j} \geq d\right\}$ means that all observations with a $j^{\text {th }}$ variable lower than $d$ are placed in the left child node. Hence, all observations with a $j^{\text {th }}$ variable greater than $d$ are placed in the right child node. The method selects the best pair $(j, d)$ which minimize a loss function. Here, we aim at minimizing the variance of the child nodes. The variance of a given node $t$ is defined as:

$$
\sum_{i: X_{i} \in t}\left(Y_{i}-\bar{Y}_{t}\right)^{2}
$$

where $\bar{Y}_{t}$ is the averaged $Y_{i}$ in the node $t$.

The same procedure is then applied recursively to the next child nodes using the same variables until a certain stop criterion is reached. The procedure automatically stops if each node contains a unique observation. Hence, the maximal depth of a regression tree is $n-1$. An illustration of such tree is presented in Fig 5

A random-input regression tree is used here. This is a particular case of regression trees, in which a set of $m<p$ variables is randomly preselected before applying the regression tree. A large number $K$ of random-input trees is computed. For each tree, we randomly select $m<p$ variables with probability $\frac{1}{p}$ and we apply the method until it reaches its maximal depth.

\subsubsection{Reconstruction}

The reconstruction is obtained by splitting each testing series in the different trees. In each tree, the estimation attributed to an observation is the empirical average of $Y$ inside the node where the corresponding observation ends up, given the cut made on the corresponding predictors. For each testing series, the $K$ reconstructions are averaged to give the final reconstruction. 


\subsubsection{Parameters}

A priori, this method requires the optimization of two parameters: the number of trees $K$ and the number of variables selected for each tree $m$. In practice $K$ does not require tuning, as long as the number of trees is sufficiently high given $\mathrm{p}$, which guarantees convergent results for any value of $m$ (Breiman, 2001). $m$ is then the only parameter to optimize. The KFCV is then applied on $m$ with a high $K$ (here set to 1000 ), to select empirically the most efficient model. 


\section{Supplementary material 2: Statistical test for correlation signifi- cance}

The statistical test we use in all the study as been firstly proposed by Bretherton et al. (1999) to avoid the individual autocorrelation effects on the correlation between two series. This is done by adjusting the degree of freedom. However, a simplification of this test has been proposed by McCarthy et al. (2015) by only using the first order autocorrelations to modify the degree of freedom.

Let $X=\left(X_{t}\right)_{t \in T}$ and $Y=\left(Y_{t}\right)_{t \in T}$ two time series of same length. The correlation between the two series is given by:

$$
r=\operatorname{cor}(X, Y)=\frac{\operatorname{cov}(X, Y)}{\sqrt{\operatorname{Var}(X)} \cdot \sqrt{\operatorname{Var}(Y)}}
$$

We denote $a_{1}^{(X)}$ and $a_{1}^{(Y)}$ the first order lag of the respective autocorrelation functions of $X$ and $Y$. The effective number of degrees of freedom (Bretherton et al., 1999) is then given by :

$$
N_{e f f}=N_{o b s} \cdot \frac{1-a_{1}^{(X)} \cdot a_{1}^{(Y)}}{1+a_{1}^{(X)} \dot{a}_{1}^{(Y)}}
$$

The statistics is then calculated as :

$$
t^{\text {stat }}=\sqrt{N_{e f f}} \cdot \frac{r}{\sqrt{1-r^{2}}}
$$

For $\alpha \in] 0,1\left[\right.$, the statistic $t^{\text {stat }}$ is compared to the $1-\frac{\alpha}{2}$ order quantile of a Student distribution with $N_{e f f}$ degrees of freedom. 


\section{Supplementary material 3: Uncertainties}

The uncertainties we provide are calculated as in Ortega et al. (2015) but using the residuals calculated for the final moel. These regression uncertainties are represented by the standard errors (s.e.) of the

regression, calculated as the root of the sum of the squared residuals over the training periods divided by the degree of freedom:

$$
s . e=\sqrt{\frac{\sum_{i=1}^{n}(Y-\hat{Y})^{2}}{n-2}}
$$

Where $n_{\text {train }}$ is the length of the learning sample, $Y$ the true values of the NAO index over the learning period, and $\hat{Y}$ the fitted NAO by the regression model over the training period. An uncertainty band $2 *$ s.e. is calculated which is our estimate of the total uncertainty range of the reconstruction. 


\section{Supplementary material 4: Superposed Epoch Analysis}

For both sets of volcanic eruptions (3.2.2), each member of the composite is extracted from year N-1 to year $\mathrm{N}+4$ from the corresponding NAO reconstruction, $\mathrm{N}$ standing for the year of the eruption. Hence, they are centred to the year of the eruption and averaged at each time step to perform the composite timeseries presented in Fig 12. The significance levels are calculated using a Monte Carlo approach as proposed by Rao et al. (2019). We randomly draw 10000 composite of 11 (for Sigl. et al, 2015, see Fig 12) and 10 (for Ortega et al, 2015, see Fig 12) N-1 to N+4 sub-timeseries of the corresponding NAO index. As for the real composite, each member of the composites is centred to the year $\mathrm{N}$ and are averaged over time. Hence, for each time step, we keep the $90^{\text {th }}$ percentile among the 10000 sorted composite values as the $90 \%$ confidence level presented Fig 12. 